\title{
Médiévales
}

Langues, Textes, Histoire

69 | automne 2015

Travailler à Paris (XIIIe-XVIe siècle)

\section{Pratiques alimentaires, savoir-faire et professionnalisme dans les métiers de bouche parisiens (fin du Moyen Âge et Renaissance)}

Food Practices, Know-How and Professionalism in the Parisian Food Trades (Late Middle Ages and Renaissance)

\section{Patrick Rambourg}

\section{OpenEdition \\ Journals}

Édition électronique

URL : https://journals.openedition.org/medievales/7601

DOI : 10.4000/medievales.7601

ISSN : $1777-5892$

\section{Éditeur}

Presses universitaires de Vincennes

\section{Édition imprimée}

Date de publication : 30 novembre 2015

Pagination : 87-104

ISBN : 978-2-84292-444-7

ISSN : 0751-2708

\section{Référence électronique}

Patrick Rambourg, «Pratiques alimentaires, savoir-faire et professionnalisme dans les métiers de bouche parisiens (fin du Moyen Âge et Renaissance) », Médiévales [En ligne], 69 | automne 2015, mis en ligne le 30 novembre 2017, consulté le 23 avril 2022. URL : http://journals.openedition.org/ medievales/7601; DOI : https://doi.org/10.4000/medievales.7601 


\section{Patrick Rambourg}

\section{Pratiques alimentaires, savoir-faire et professionnalisme dans les métiers de bouche parisiens (fin du Moyen Âge et Renaissance)}

Dans un bel article intitulé « Métiers licites et métiers illicites dans l'Occident médiéval ${ }^{1}$ », Jacques Le Goff montre comment nombre de professions ${ }^{2}$ ont longtemps été méprisées, à l'exemple des métiers de l'alimentation. C'est notamment le cas des bouchers, en raison du tabou du sang, et des métiers qui touchent à l'un des sept péchés capitaux : les aubergistes, les taverniers et les tenanciers d'étuves, condamnés pour luxure, ou les cuisiniers, pour gourmandise, qui étaient également taxés d'impureté et de saleté. Car, affirme Adalbéron de Laon vers l'an mille, les clercs exempts de travaux serviles « ne sont ni bouchers, ni aubergistes [...], ignorent la cuisante chaleur d'une marmite graisseuse [...], ils ne sont pas blanchisseurs et dédaignent de faire bouillir le linge ${ }^{3} »$.

Tout cela valait pour le haut Moyen Âge dans une société occidentale largement rurale, où le mépris touchaitun grand nombre d'activités manuelles qui n'étaient pas liées directement à la terre. Mais la situation évolua au cours des $\mathrm{XII}^{\mathrm{e}}$ et $\mathrm{XIII}^{\mathrm{e}}$ siècles avec l'essor urbain et la reconnaissance du travail. Dans la société, « chaque profession a [désormais] son rôle matériel et sa valeur spirituelle. Aucun métier n'est un obstacle au salut, chacun a sa vocation chrétienne, chacun rejoint cette "familia Christi" qui groupe tous les bons travailleurs », écrit Jacques Le Goff ${ }^{4}$. L'Église, qui avait vu avec suspicion les professions s'organiser en corps, finit par accepter cette

1. J. LE GoFf, Pour un autre Moyen Âge, Paris, 1977, p. 91-107.

2. Le terme «professionnalisme » utilisé dans le titre s'entend au sens de compétences techniques et spécifiques qui se transmettent par apprentissage au sein d'un métier. Cela suggère à la fois la connaissance du métier exercé, la conscience professionnelle de la personne qui le pratique, et le travail bien fait. Ce qui en même temps contribue à définir l'identité d'une profession.

3. J. LE GofF, Pour un autre Moyen Âge..., p. 94.

4. Ibid.,p. 103. 
évolution : les gens de métier constituent dès lors « un autre secteur légal, un groupe social aux droits reconnus ${ }^{5} »$. Ils participent à l'embellissement des édifices religieux en finançant notamment des vitraux qui représentent leurs activités professionnelles, comme en témoignent les verrières de la cathédrale de Chartres avec les bouchers, les boulangers, les épiciers et les marchands de poissons ${ }^{6}$.

Les corps de métiers deviennent dès lors une force que les pouvoirs publics ne peuvent plus ignorer. C'est particulièrement vrai des métiers de l'alimentation qui s'imposent dans la capitale, jusqu'à occuper, selon Jean Favier, le premier rang des métiers de la ville quant au nombre de leurs représentants ${ }^{7}$. Certes, la quantité de bouches à nourrir contribue au développement de ces métiers, dont la prospérité est "étroitement liée à l'importance quantitative du marché de consommation ${ }^{8} »-$ la ville de Paris, malgré les crises successives, passe de deux cent mille à trois cent mille habitants entre le $\mathrm{XIV}^{\mathrm{e}}$ et le $\mathrm{XVI}^{\mathrm{e}}$ siècle - mais ces professionnels de l'alimentation surent se rendre indispensables en affirmant leurs spécificités et en jouant un rôle important auprès des populations modestes, sans toutefois négliger la clientèle aisée.

Plus encore, ils réussissent à s'imposer dans la longue durée en se spécialisant dans un domaine précis, en affinant leur savoir-faire et la technicité de leurs professions respectives. C'est précisément le cas des spécialistes du " prêt-à-manger ${ }^{9}$ », c'est-à-dire de ceux qui apprêtent et confectionnent des mets prêts à être consommés, sur lesquels nous nous arrêterons plus particulièrement. Ils contribuent également à créer des habitudes de consommation auprès des Parisiens qui font appel à leurs services ${ }^{10}$. Les textes réglementaires, qui constituent l'essentiel de nos sources, permettent d'appréhender ces professionnels de l'alimentation dans leur contexte historique, de suivre leurs préoccupations et d'entrevoir la manière dont ils pensent leur métier.

5. É. COORNAERT, Les Corporations en France avant 1789, Paris, 1941, p. 63.

6. J. SAuvanon, Les Métiers au Moyen Âge. Leurs "signatures» dans les vitraux. Cathédrale de Chartres, Chartres, 1993.

7. J. FAVIER, Paris au XVe siècle, Paris, 1997, p. 304.

8. Ibid., p. 304.

9. P. RAMBOURG, « Des spécialistes du prêt-à-manger à Paris : cuisine publique et modes de restauration à la fin du Moyen Âge », dansV. MARCILHAC et V. MORINIAUX éd., Les Établissements de restauration dans le monde, Paris, 2012, p. 113-125.

10. Dans leurs statuts, les « cuisiniers-oyers » disent apprêter des « viandes communes » pour le «peuple» (Le Livre des métiers d'Étienne Boileau, éd. R. DE LESPINASSE et F. BonNARDOT, Paris, 1879, p. 145). En juillet 1513, les charcutiers évoquent, dans leurs statuts, le «pauvre menu peuple », les « forains qui affluent chascun jour », les « bourgeois de ladite ville », les « bonnes maisons » (Lettres patentes de Louis XII portant confirmation des statuts des charcutiers (18 juillet 1513), dans Les Métiers et corporations de la ville de Paris, éd. R. De LeSPINASSE, Paris, 1886, t. I, p. 324-325). 
Au temps de Saint Louis, la plupart des statuts professionnels de la capitale sont consignés dans Le Livre des métiers ${ }^{11}$. Ce célèbre recueil présente un état des lieux qui permet de classifier les professions de bouche de l'époque, et donne un point de départ pour suivre leurs mutations dans les sources réglementaires édictées dans la suite du Moyen Âge et à la Renaissance. C'est une longue tradition d'organisation professionnelle et d'intervention des pouvoirs politiques qui se dessine, où l'interaction entre l'intention normative de la puissance publique et la volonté d'organisation et de codification des corps de métier eux-mêmes montre des artisans construisant leur identité professionnelle. Ceux-ci définissent les contours de leur spécialisation et de leur domaine de compétence par la pratique et la connaissance du métier, tout en suivant les évolutions de la société et des goûts alimentaires.

\section{Les métiers de l'alimentation dans le recueil d'Étienne Boileau}

Dès le XIII ${ }^{\mathrm{e}}$ siècle, les professions organisées sont « choses urbaines $^{12} »$. Le Livre des métiers (1268) du prévôt de Paris, Étienne Boileau, représentant du roi, est une source essentielle à la compréhension des métiers parisiens. Il regroupe une centaine de corps professionnels qui ont dit et enregistré leurs statuts au tribunal du Châtelet. Il découle de la volonté du roi et de celle des métiers qui viennent eux-mêmes déclarer leurs règlements. Cette mise par écrit des usages et des coutumes est une reconnaissance officielle des gens de métiers. Ces derniers mettent en avant leur préoccupation du moment dans un Paris en pleine prospérité, ce qui nuance du même coup l'idée d'un « schéma commun à tous les statuts ${ }^{13}$ » : l'historien peut dès lors toucher une réalité au-delà du texte réglementaire.

Par la rédaction de statuts, les métiers trouvent un moyen de renforcer ou de créer un monopole professionnel, c'est-à-dire se réserver l'exclusivité de la confection de produits spécifiques, pour protéger et valoriser les savoirfaire et les techniques, et ainsi préserver de la concurrence et du chômage ceux qui exercent cette activité ${ }^{14}$. De leur côté les autorités royales policent les métiers de la capitale et les placent sous leur tutelle, notamment les

11. Ou « Li establissement des mestiers de Paris ». Il y a deux éditions principales du Livre des métiers : Réglemens sur les arts et métiers de Paris, rédigés au XIII siècle, et connus sous le nom du livre des métiers d'Étienne Boileau, éd. G. B. DEPPING, Paris, 1837 ; et Le Livre des métiers d'Étienne Boileau, éd. R. DE LESPINASSE et F. BONNARDOT, Paris, 1879. Je m'appuierai sur cette dernière édition désormais désignée comme Le Livre des métiers.

12. É. COORNAERT, Les Corporations..., p. 71.

13. S. Roux, Paris au Moyen Âge, Paris, 2003, p. 168.

14. R. CAZElles, Paris de la fin du règne de Philippe Auguste à la mort de Charles V, Paris, 1994, p. 86. 
métiers de bouche, afin de mieux contrôler l'approvisionnement de la ville et la sécurité alimentaire du citadin ${ }^{15}$. Mais tous les métiers ne déposèrent pas leurs statuts devant le prévôt de Paris ${ }^{16}$. Il en va ainsi - c'est le cas le plus connu -, des bouchers, pourtant l'un des corps professionnels les plus anciennement réglementés, que l'on a dit de filiation antique ${ }^{17}$.

Les métiers touchant à l'alimentation présentés dans le recueil d'Étienne Boileau peuvent être classés en cinq catégories ${ }^{18}$. Il y a ceux qui proposent des denrées non apprêtées que l'on pourrait qualifier de « matières premières »: ce sont les «blaetiers » qui vendent du blé et toutes sortes de grains ; les meuniers du «Grand Pont » qui disposent de moulins leur permettant de moudre les grains ; les volaillers qui vendent des volatiles chaque jour de l'année à la Porte de Paris et en la rue Neuve Notre-Dame, et aux halles en Champeaux le samedi. Il y a aussi les pêcheurs-poissonniers qui forment trois communautés : les «Pescheurs de l'eaue le Roy ${ }^{19}{ }^{»}$, les poissonniers d'eau douce et les poissonniers de mer. Cela montre l'importance du poisson dans le commerce alimentaire de l'époque ${ }^{20}$, importance liée aux prescriptions du calendrier chrétien que personne ne pouvait ignorer. L'alternance des jours maigres (à poisson) et

15. P. RAmbourG, « Les savoirs alimentaires dans le Paris de la fin du Moyen Âge : entre pratique culinaire et hygiène alimentaire », dans M. BRUEGEL, M. NICOUD et É. BARLÖSIUS éd., Le Choix des aliments. Informations et pratiques alimentaires de la fin du Moyen Âge à nos jours, Rennes/Tours, 2010, p. 181-196.

16. Notamment les marchands de l'eau, les bouchers, et certains métiers du cuir : É. COORNAERT, « Notes sur les corporations parisiennes au temps de saint Louis. D'après le Livre des métiers d'Étienne Boileau », Revue historique, 177 (1936), p. 344.

17. «Une corporation parisienne qui se vantoit d'une origine très ancienne, étoit celle des bouchers. Ce qui prouve en effet son antiquité, c'est qu'elle avoit conservé quelque chose de l'organisation donnée sous les empereurs romains aux corporations des bouchers dans les villes », raconte G. B. DEPPING dans Réglemens sur les arts et métiers de Paris..., p. LIV. Émile COORNAERT nuance cette affirmation en écrivant au sujet des bouchers : « dont certains [auteurs] voulurent faire remonter les organisations - mais à travers quelles vicissitudes ? jusqu'aux collegia gallo-romains » (« Notes sur les corporations parisiennes... », p. 345).

18. Dans leur introduction au Livre des métiers, René De LESPINASSE et François BONNARDOT classent les métiers concernant l'alimentation en trois catégories : «farines », « boissons », «épicerie et vivres en général » (Le Livre des métiers..., p. XIX-XXXVIII). Il m'a paru nécessaire de proposer un classement différent, en fonction du degré d'élaboration des produits, depuis les denrées brutes jusqu'aux aliments apprêtés.

19. L'eau du roi concernait la Seine et la Marne, « depuis la pointe de l'île Notre-Dame jusqu'à Saint-Maur-des-Fossés » à l'est de Paris. Les poissons pêchés approvisionnaient les cuisines de la cour : la dimension des mailles des filets était contrôlée par le maître queux du roi pour ne prendre qu'une catégorie de poissons (brochets, barbeaux, anguilles et carpes). Les cuisiniers du roi avaient aussi un droit de prise sur la pêche des poissonniers d'eau douce (seconde communauté) et choisissaient les meilleurs poissons pour la table royale (Le Livre des métiers..., p. XXXV et 212).

20. C. Bourlet, « L'approvisionnement de Paris en poisson de mer aux XIV et $\mathrm{XV}^{\mathrm{e}}$ siècles, d'après les sources normatives », Franco-British Studies. Journal of the British Institute in Paris, 20 (1995), p. 5-22. 
des jours gras (à viande), en plus des périodes de jeûne du Carême et de l'Avent, marque alors profondément les modes de consommation ${ }^{21}$.

Les mesureurs de denrées forment le deuxième groupe des métiers liés à l'alimentation. Ces sortes d'officiers publics, qui relèvent de la Prévôté des marchands, ne confectionnent aucun produit, ne vendent aucune marchandise, ne disposent ni d'atelier ni de boutique, mais constituent des intermédiaires indispensables « entre les marchands et les acheteurs, pour le prix, la qualité, le mesurage 22 ». Leur intervention n'est pas obligatoire et l'on fait généralement appel à eux en cas de litige. Il y a les « mesureurs de blé et de toute autre manière de grain » et les « jaugeurs » préposés aux liquides, dont le miel.

La troisième catégorie de métiers regroupe les « cervoisiers », les taverniers et les crieurs, c'est-à-dire ceux qui fabriquent, débitent et vendent des boissons. Les cervoisiers confectionnaient leur cervoise avec de l'eau et des céréales, principalement de l'orge, du méteil (mélange de blé et seigle), et autres grains concassés et fermentés que l'on appelait «dragie ». Les statuts préconisent la vente sur le lieu de fabrication et déconseillent le déplacement, arguant du risque d'une cervoise « aigre et tournée ». Les taverniers sont avant tout des vendeurs de vins au détail ${ }^{23}$. Ils doivent s'adjoindre les services d'un crieur chargé d'annoncer le prix du vin dans la rue au nom du commerçant. Le crieur et le tavernier s'accordent sur le tarif du vin à crier. Le crieur s'assure de sa qualité en regardant tirer le vin ou en le tirant lui-même du tonneau, puis va dans la rue avec un pot de vin et un hanap pour pouvoir le faire goûter au client potentiel ${ }^{24}$. Crier les marchandises dans les rues des villes est alors chose courante. Les Crieris de Paris $^{25}$, forme littéraire qui apparaît au XIII ${ }^{\mathrm{e}}$ siècle, évoquent toutes sortes de denrées alimentaires vendues dans la rue, comme le font entendre encore

21. Voir à ce sujet C. LAMBERT, « La nourriture comme signe de distinction religieuse et sociale de Thomas d'Aquin à Érasme », Heresis, 26-27 (1996), p. 99-113 ; B. LAURIOUX, Manger au Moyen Âge, Paris, 2002, p. 101-122.

22. Le Livre des métiers..., p. XXVI.

23. D'ailleurs toute personne qui avait du vin à vendre pouvait le faire à taverne, dans son cellier, chez lui ou devant sa porte. Il lui suffisait de payer un impôt spécifique appelé « chantelage ».

24. Le vin était crié deux fois dans la journée (matin et midi), sauf certains jours de fêtes religieuses où il n'était crié qu'une fois, voire pas du tout, art. XII : « Li Crierres doit crier chascun jour II foiz, fors mis le Quaresme, les diemenges, les vendredis, et les VIII jourz de Nouel, et les Vigiles, qu'il ne crient que une foiz ; le vendredi de Croiz Aourée [Vendredi de la croix adorée, le Vendredi saint] ne crient pas Crieurs, mès il encusent après le service ». On ne le criait pas non plus lors du décès d'un membre de la famille royale (Le Livre des métiers..., p. 23).

25. A. Franklin, Les Rues et les cris de Paris au XIII siècle, Paris, 1984, p. 153-164. 
dans la musique de la Renaissance les cris de Paris de Clément Janequin $(1480-1560)^{26}$.

La quatrième catégorie est celle des regrattiers, qui forment deux corps professionnels dans Le Livre des métiers ${ }^{27}$. Revendeurs de comestibles et de vivres au détail, ils jouent un rôle important en offrant une nourriture du quotidien. Selon les statuts des deux communautés, les regrattiers proposent du poisson de mer, de la viande cuite, des œufs, du fromage, des volatiles, des fruits comme les pommes, les raisins, les figues et les dattes, des herbes potagères, des alliacés comme l'ail, l'échalote et l'oignon, du sel, et quelques épices à l'exemple du poivre, du cumin et de la cannelle.

La cinquième catégorie de notre classement concerne les professions apprêtant des aliments. Les huiliers fabriquent des huiles d'olives, d'amandes, de noix, de chènevis et de pavots. Les " cuisiniers-oyers » cuisinent et rôtissent des oies, de la viande de veau, d'agneau, de chevreau, de porc, et préparent des saucisses. Ils apportent des conseils sur la qualité de la viande et sur sa conservation qui ne doit pas dépasser trois jours lorsqu'elle est cuite. Ils interdisent la vente du boudin de sang, une «périlleuse viande », suggérant par là l'existence de mauvaises pratiques et révélant en même temps une préoccupation naissante de la sécurité alimentaire des consommateurs, ou du moins de la «perception du risque alimentaire $^{28} »$. Puis il y a les boulangers que l'on appelle «talemeliers ». Leur statut ouvre Le Livre des métiers. C'est, du reste, avec ses soixante et un articles, l'un des plus longs du recueil (avec ceux des tisserands et des fripiers). Cela montre l'ancienneté de la profession ${ }^{29}$, mais surtout l'importance du pain dans l'alimentation médiévale ${ }^{30}$. Outre les détails sur le poids et le prix du pain, dont nous reparlerons plus loin, on apprend que les boulangers ne peuvent faire de cuisson le dimanche et certains jours de fête ${ }^{31}$, que le pain se vend en boutique les jours de la semaine, et que

26. «Voulez ouyr les cris de Paris », dans Les Cris de Paris. Chansons de Janequin \& Sermisy. Ensemble Clément Janequin, D. Visse, Arles, Harmonia mundi, 2005.

27. « Des Regratiers de pain, de sel, de poisson de mer et de toutes autres denrées, fors poisson de eaue douce et cire ouvrée ", "Cis titres parole des Regratiers qui vendent fruit et aigrun a Paris » (Le Livre des métiers..., p. 27-32).

28. Selon Madeleine Ferrières, la perception du risque alimentaire avait deux versants : l'un «portait sur la quantité et la régularité de l'approvisionnement », l'autre « concernait la salubrité des aliments » (Histoire des peurs alimentaires du Moyen Âge à l'aube du XXe siècle, Paris, 2002, p. 11-12). Voir M. BRUEGEL et A. STANZIANI, « Pour une histoire de la "sécurité alimentaire" », Revue d'histoire moderne et contemporaine, 51-3 (2004-3), p. 7-16.

29. L'article XX des statuts se réfère à Philippe Auguste (1165-1223). «Cis titres parole des Talemeliers qui sunt dedens la banlieu de Paris » (Le Livre des métiers..., p. 3-15).

30. Voir à ce sujet F. DesPortes, Le Pain au Moyen Âge, Paris, 1987.

31. Quatre-vingts jours environ, soit presque le quart de l'année : Le Livre des métiers..., p. 8. 
le samedi est réservé aux boulangers forains, qui viennent des villages environnants proposer leur pain dans les marchés de la ville.

Ainsi les métiers de l'alimentation sont déjà bien codifiés dans le Paris de la fin du XIII ${ }^{\mathrm{e}}$ siècle. Le Livre des métiers s'ouvre avec des professions de bouche, suggérant peut-être leur importance dans la ville : les dix premiers sont les talemeliers, les meuniers du Grand-Pont, les blatiers, les mesureurs de blé, les crieurs de vins, les jaugeurs, les taverniers, les cervoisiers, les regrattiers de pain et de sel, les regrattiers de fruits et de légumes. Mais en même temps, les cuisiniers et les poulaillers arrivent respectivement à la $69^{\mathrm{e}}$ et la $70^{\mathrm{e}}$ position, tandis que les pêcheurs et les poissonniers occupent les trois dernières places de l'ordonnancement des métiers. Les règlements présentés dans le Livre des métiers concernent pour l'essentiel les matières premières, la vente et l'apprêt d'une nourriture du quotidien. Ils reflètent une réalité parisienne, corroborée par d'autres sources ${ }^{32}$, dans laquelle le citadin, mais aussi le voyageur, a à sa portée une alimentation facile à acquérir et prête à consommer. Les statuts de métier couchés dans le recueil du prévôt de Paris ont par la suite été complétés et sont devenus des textes de référence pour la rédaction de nouveaux règlements ${ }^{33}$.

\section{Vers une plus grande spécialisation des métiers de bouche}

Aux XIV et $\mathrm{XV}^{\mathrm{e}}$ siècles apparaissent de nouveaux corps de métiers ${ }^{34}$ et un plus large éventail de professions de bouche. Le processus suit un triple mouvement. Le premier donne naissance à des communautés dans

32. Certaines de ces sources sont citées dans notre propos, comme par exemple : les Crieries de Paris (XIII ${ }^{\mathrm{e}} \mathrm{s}$.), le Dictionarius de Jean de Garlande (XIII $\left.{ }^{\mathrm{e}} \mathrm{s}.\right)$, Le Mesnagier de Paris (vers 1393), le Journal d'un bourgeois de Paris (XIV ${ }^{\mathrm{s}}$ s.). Nous pourrions également évoquer les comptabilités, comme celle de la confrérie Saint-Jacques-aux-Pèlerins : $c f$. P. RAMBOURG, «Les repas de confrérie à la fin du Moyen Âge : l'exemple de la confrérie parisienne Saint-Jacques-aux-Pèlerins au travers de sa comptabilité (XIVe siècle) », dans F. RAVOIRE et A. DiETRICH éd., La Cuisine et la table dans la France de la fin du Moyen Âge : contenus et contenants du XIV au XVI siècle, Caen, 2009, p. 51-78. Ou encore la comptabilité de l'Hôtel-Dieu : $c f$. C. JÉHANNO, « L'alimentation hospitalière à la fin du Moyen Âge : l'exemple de l'Hôtel-Dieu de Paris », dans G. Drossbach éd., Hospitäler in Mittelalter und Früher Neuzeit. Frankreich, Deutschland und Italien. Eine vergleichende Geschichte. Hôpitaux au Moyen Âge et aux Temps modernes. France, Allemagne et Italie. Une histoire comparée, Munich, 2007, p. 107-162.

33. Étienne Boileau confiera à ses successeurs « le soin de corriger ou d'augmenter les statuts, à la demande des maîtres et des jurés ». Et les boulangers resteront fidèles à leur statut du Livre des métiers jusqu'en 1719 (Les Métiers et corporations..., p. I et 195).

34. La création de ces nouveaux corps de métier ne veut pas dire pour autant que l'activité professionnelle n'existait pas avant. C'est ainsi le cas des pâtissiers dont nous trouvons trace bien avant la constitution de leur statut en 1440, comme je le signale dans la suite de mon propos. 
lesquelles les artisans se regroupent selon leurs spécialisations culinaires et alimentaires, à l'exemple des charcutiers et des rôtisseurs, les uns se spécialisant dans l'apprêt du porc, les autres dans les viandes rôties. Le second mouvement est dans la disparition de petits métiers qui sont généralement intégrés dans un corps professionnel plus puissant ; c'est notamment le cas des fabricants de gaufres et de gâteaux, absorbés par les oubloyers qui seront eux-mêmes absorbés par les pâtissiers au XVI ${ }^{\mathrm{e}}$ siècle. Enfin, le troisième mouvement montre des métiers qui n'ont en apparence aucun lien particulier, mais qui se regroupent ou sont réunis dans une même communauté, tels les « vinaigriers-sauciers-moutardiers » de 1394, dont le vinaigre semble être l'élément commun ${ }^{35}$.

Les textes réglementaires ${ }^{36}$ continuent à porter sur l'organisation des métiers, mais se font aussi plus concrets sur le savoir-faire alimentaire et la pratique culinaire. Ils montrent l'importance grandissante que les professionnels accordent à la technique, à la connaissance des produits, à la qualité et à la confection des recettes. Les premiers statuts des boulangers (talemeliers), ceux du Livre des métiers, distinguent trois sortes de pains : le pain dit demie d'une obole, «le pain d'un denier (la denrée) » et le pain de deux deniers ou doubleau ${ }^{37}$. Mais ces différences correspondent à des différences de taille et non de qualité ${ }^{38}$. On considérait qu'un pain était «bon » lorsqu'il était fait avec une farine « loyale » et qu'il avait la dimension requise ${ }^{39}$. Le consommateur acquérait un pain à prix fixe, dont le poids pouvait varier selon le tarif du blé. Le citadin recevait ainsi pour une même somme « un pain plus ou moins lourd selon les jours ${ }^{40} »$.

C'est au cours du XIV siècle que l'on commence à établir un rapport entre la valeur du blé et la qualité du pain. Cette évolution n'est pas spécifique à la capitale et se rencontre aussi dans d'autres villes ${ }^{41}$. La grande ordonnance de Jean II le Bon (1351) «sur la police générale et sur

35. «Ordonnance de Jehan de Folleville, prévôt de Paris, contenant les premiers statuts des vinaigriers-sauciers-moutardiers $»(28$ octobre 1394) (Les Métiers et corporations..., p. 568).

36. Il s'agit de règlements édictés ponctuellement selon l'évolution de chacun des métiers, même s'il existe des textes touchant l'ensemble des professions, comme la grande ordonnance de Jean II le Bon de 1351. La plupart ont été rassemblés dans Les Métiers et corporations... ou encore dans N. Delamare, Traité de la Police, Paris, 1710, t. II.

37. Le Livre des métiers..., p. XXII.

38. G. FAGNIEZ, Études sur l'industrie et la classe industrielle à Paris au XIII et au XIV siècle, Genève/Paris, 1975, p. 174.

39. Art. XXXIII des statuts des talemeliers : «Tout li Talemelier doivent faire denrées et demies et pains de II d. bons et loiaus, selonc le marchié qu'il ont du blé » (Le Livre des métiers..., p. 9).

40. «Les plus démunis trouvaient dans la taxation d'un aliment de première nécessité [le pain] une sécurité, illusoire sans doute, mais qui allégeait leur crainte viscérale du lendemain » (F. DESPORTES, Le Pain..., p. 145-149).

41. Ibid., p. 149 sq. 
les divers métiers de la ville de Paris ${ }^{42}{ }$ fixe ainsi le prix de la pâte et du pain selon le cours du blé, qui varie de 24 à 40 sous le setier ${ }^{43}$. Le titre II de l'ordonnance, consacré aux blés, aux pains et aux boulangers, donne la concordance entre l'ancienne distinction du pain, établie selon son prix, et la nouvelle distinction, fondée sur des différences de qualité. Le pain n'est plus seulement perçu comme une denrée de nécessité, mais aussi comme un aliment dont on peut apprécier le goût, tout en étant socialement marqué ${ }^{44}$.

Il y avait trois sortes de pain selon le degré de blutage de la farine : le pain de « Chailly ${ }^{45}$ », qui était blanc et fait à l'imitation de celui que l'on faisait dans un village des environs de Paris ; le pain « coquillé », dont la croûte formait des sortes de boursouflures, et que l'on appellera plus tard pain bourgeois (d'une couleur plus ou moins jaune) ; enfin le pain « faitis » ou « de brode », à base de farine complète ${ }^{46}$. On retrouve ces trois variétés de pain citées dans le Journal d'un bourgeois de Paris, à l'année $1419:$ « [...] et que chacun boulanger fît bon pain blanc, pain bourgeois et pain festiz à toute sa fleur, et de certain poids dit au $\mathrm{cri}^{47} »$. Le pain « de chapitre » est une quatrième variété de pain qui apparaît dans le courant du $\mathrm{XVI}^{\mathrm{e}}$ siècle ${ }^{48}$. Il était aussi blanc que le pain de «Chailly », mais composé d'une pâte plus affermie ${ }^{49}$.

Dans la manière de faire le pain, il existait aussi des différences entre les boulangers parisiens et ceux des faubourgs. Les premiers trempaient plus

42. «Ordonnance du roi Jean II, sur la police générale et sur les divers métiers de la ville de Paris »(30 janvier 1351), titre 2 (Les Métiers et corporations..., p. 3-6).

43. Il y a eu un premier essai le vendredi avant la Pentecôte 1316, mais la mort du roi, peu de jours après, arrêta l'initiative (N. Delamare, Traité de la Police..., p. 894). Il faudra cependant attendre la fin des années 1430 pour que soit définitivement fixé le poids du pain. Ce ne sera plus le poids mais le prix du pain qui variera désormais selon le cours du blé : « Lettres patentes de Charles VII contenant des règlements pour les boulangers et les meuniers, sur le poids et le prix du pain à Paris » (19 septembre 1439) (Les Métiers et corporations..., p. 204-207).

44. La couleur du pain sera longtemps un élément de distinction sociale, le pain blanc trouvant sa place sur la table des élites : $c f$. M. MONTANARI, La Faim et l'abondance. Histoire de l'alimentation en Europe, Paris, 1995, p. 49.

45. Gustave Fagniez parle de pain de « Chilly » et fait le lien avec Chilly-Mazarin : cf. G. FAGNIEZ, Études sur l'industrie..., p. 176.

46. F. DESPORTES, Le Pain..., p. 96.

47. Journal d'un bourgeois de Paris, éd. C. BEAUne, Paris, 1990, p. 140.

48. «Reglement general de Police arresté au Conseil du Roy le 4 février 1567 » (N. Delamare, Traité de la Police..., p. 907-908).

49. «Une quatrième espece de pain aussi blanc que le pain de Chailli ; mais avec cette difference que celui-cy estoit molet, \& que la paste de celui-là estoit affermie, \& pour ainsi dire broyée avec tant de force que les bras n'y pouvant pas suffire, les Boulangers y employoient les pieds, après se les estre beaucoup lavez en eau chaude. On le nomma pain de Chapitre, parce que ce fut le Boulanger du Chapitre de Nostre-Dame qui en fit le premier » (N. Delamare, Traité de la Police..., p. 893). 
leur pâte et la cuisaient moins que les seconds ${ }^{50}$. Les boulangers parisiens devaient cuire leurs pains « à heure competante, tellement que iceux pains chacun en leur qualité soient froids \& rassis à heure raisonnable ${ }^{51}$ », « à sçavoir du moins pour le disner à six à sept heures du matin ${ }^{52} »$. Le bon pain était celui qui avait été « bien labouré, fermenté \& boulangé ». Certains pains de la banlieue étaient réputés, comme ceux de Corbeil, commune située à la confluence de l'Essonne et de la Seine, où se trouvaient des moulins fariniers, et dont les boulangers produisaient un pain qu'ils allaient vendre le samedi aux halles de Paris ${ }^{53}$. Celui de Saint-Brice était également fort apprécié, comme en témoigne Le Journal d'un bourgeois de Paris en 1414 : «Et advint ce samedi devant, que ceux qui amenaient les biens à Paris, comme le pain de Saint-Brice, comme autres biens et vivres ${ }^{54} »$. Et dès la fin du XV $\mathrm{XV}^{\mathrm{e}}$ siècle, le pain de Gonesse, village proche de la capitale, acquiert une réputation durable avec une recette spécifique qui en fera le succès ${ }^{55}$.

Longtemps les boulangers confectionnèrent des petits pâtés qui deviendront l'apanage des pâtissiers lorsque ceux-ci déposeront leurs statuts en $1440{ }^{56}$. Ces derniers pouvaient ainsi préparer des pâtés de viande ou de poisson, des flans, des darioles, des tartelettes au fromage, des rissoles, etc. L'existence des pâtissiers est attestée dès le début du XIII ${ }^{\mathrm{e}}$ siècle dans le Dictionarius de Jean de Garlande ${ }^{57}$, dans la grande ordonnance de $1351^{58}$,

50. « [...] et si dit [un boulanger de Notre-Dame-des-Champs près Paris] que les boulengiers de Paris abreuvent leur pain beaucop plus que lui ne les autres boulengiers des faulxbourgs, c'est assavoir de bien ung seau d'eaue plus sur le sextier et cuisent plus leur pain que lesd. boulengiers de Paris » (Livre du Châtelet Rouge, $3^{\mathrm{e}}$, Paris, AN, Y3, fo $67 \mathrm{v}^{\circ}$, cité par G. FAGNIEZ, Études sur l'industrie..., note 2, p. 173).

51. «Arrest du Parlement du 16 juillet 1511 pour la Police du Pain, \& qui ordonne que les Boulangers seront visitez par le Procureur du Roy \& par les Commissaires au Chastelet » (N. Delamare, Traité de la Police..., p. 903-904).

52. «Ordonnance du Chastelet pour la Police du Pain, du 23 novembre 1546 » (N. Delamare, Traité de la Police..., p. 905-906).

53. F. DESPORTES, Le Pain..., p. 132.

54. Journal d'un bourgeois de Paris..., p. 72. Le pain de Saint-Brice arrivait par les portes nord de la ville.

55. «La recette originale consistait à introduire les gruaux dans la fleur de farine après les avoir malaxés et gonflés d'eau tiède. Selon M. ARPIN, Historique de la Meunerie et de la Boulangerie, t. II, chap. V, elle aurait été mise au point à Senlis dans le courant du XIV siècle. Malheureusement Arpin ne cite pas ses sources », écrit F. DESPORTES, Le Pain..., p. 132 et 134, n. 7.

56. « Lettres du prévôt de Paris, contenant un nouveau texte de statuts en dix-sept articles, pour les pâtissiers »(4 août 1440) (Les Métiers et corporations..., p. 376-379).

57. «Pastillarii lucrantur quam plurimum, vendendo cunctis pastillos de carnibus porcinis et pullinis, et de anguillis cum pipere, exponendo tartas, et flacones fartos caseis mollibus et ovis sanis et, quandoque, immundis » («Dictionnaire de Jean de Garlande », édité dans H. GÉRAud, Paris sous Philippe le Bel d'après des documents originaux, Paris, 1837, p. 593).

58. «Item, lesdits pasticiers ne pourront garder leurs pastez que ung jour, ne la chair de quoy ilz feront vieulx pastez, sur peine de xx sols parisis d'amende » (Ordonnance du roi Jean II..., titre 3, Les Métiers et corporations..., p. 7). 
mais aussi dans Le Mesnagier de Paris (vers 1393) où l'auteur suggère à sa jeune épouse de faire appel aux services d'un pâtissier pour la confection des pâtés : «Puis porte l'en au pasticier qui les met IIII et IIII ou III en ung pasté, et de la pouldre fine dessus ; et sans cuire ou four sont cuiz en sain ${ }^{59}$. »

Les pâtissiers étaient bien connus des Parisiens à qui ils vendaient une nourriture prête à consommer. Mais ils avaient une mauvaise réputation et étaient suspectés d'employer des ingrédients de basse qualité dans leurs préparations, et même de vendre des aliments « non dignes de user au corps humain » disait-on ${ }^{60}$. C'est un problème qui ne cessera de les préoccuper et qu'ils chercheront à combattre, en même temps que les pouvoirs publics, en prohibant l'utilisation de produits corrompus, puants ou moisis, en imposant des délais de conservation pour les pâtés - généralement pas plus d'une journée -, puis en instaurant un meilleur contrôle de la profession, en faisant notamment de celle-ci un métier juré ${ }^{61}$.

Mais la récurrence des interdits dans les règlements laisse supposer une prise de conscience plus lente que rapide. Le contrôle de la vente ambulante semblait difficile, puisque les textes de la fin du $X v^{e}$ siècle rappellent encore aux pâtissiers d'employer leurs apprentis pour porter parmi la ville les « menus ouvrages dudit métier ${ }^{62}$ », et non d'autres gens nommés «larrons », « coupeurs de bourses » et « inhabiles ». Au fil du temps, le corps des pâtissiers réussit néanmoins à transformer son image, en diversifiant d'abord ses préparations culinaires : « flannetz » (flans), tartelettes, « ratons $^{63} »$, « cassemuseaulx ${ }^{64} »$, tartes aux pommes,

59. Au sujet des pâtés norrois qui sont faits avec du foie de morue et parfois du poisson haché, voir Le Mesnagier de Paris, éd. G. BRERETON et J. FERRIER, trad. et notes K. UELTSCHI, Paris, 1994, p. 738.

60. «Sentence du prévôt de Paris prescrivant aux pâtissiers de n'avoir qu'une boutique, de ne faire colporter que par les apprentis seulement, et de ne pas assister sans droit aux festins de confrérie » (12 octobre 1489) : « [...] comme encores font de present, comporter lesdites denrées par plusieurs genz non alouez et mauvès garçons incongnus, lesquels vendent lesdites denrées secretement à pouvres gens et petis enfans, combien qu'elles soient le plus souvent incertaines et non dignes de user au cors humain » (Les Métiers et corporations..., p. 382-383)

61. Voir à ce sujet H. HAUSER, Ouvriers du temps passé (XVe-XVI siècles), Genève/Paris, 1982 (réimpression de l'édition de Paris, 1899).

62. «Lettres de Jacques d'Estouteville, prévôt de Paris, contenant une requête des pâtissiers, avec sept articles de statuts » (6 juin 1497) (Les Métiers et corporations..., p. 383).

63. Une sorte de pâtisserie faite avec du lait et des œufs : $c f$. F. GODEFrOY, Dictionnaire de l'ancienne langue française et de tous ses dialectes du IXe au XVe siècle, Paris, t. VI, 1889, p. 614.

64. Une pâtisserie croquante et dure : $c f$. F. GODEFROY, Dictionnaire de l'ancienne langue..., t. IX, 1898, p. 5. 
« tallemouses ${ }^{65}$ », échaudés ${ }^{66}$ (1497), petits choux, brioches et pain d'épices (1566) ; en taxant dans les années 1520 le prix des pâtés à 5 deniers pour assurer au consommateur la qualité des ingrédients employés dans sa fabrication, puis en contrôlant l'achat des matières premières, comme le blé, le fromage de Brie, les œufs et le beurre ${ }^{67}$.

D'autres artisans, spécialisés dans un domaine spécifique, en viennent à sortir du corps des pâtissiers pour créer leur propre communauté, à l'exemple des « fabricants de pain d'épices » qui reçoivent leurs premiers statuts en $1596^{68}$. Un arrêt du Parlement de 1508 les désigne tout d'abord du nom de «pâtissiers sucrés » (pistores dulciarii) pour les distinguer des pâtissiers de graisse et de viandes ${ }^{69}$. Le XVI ${ }^{\mathrm{e}}$ siècle est une période où le goût sucré est à la mode ${ }^{70}$. Ces pistores dulciarii confectionnent des pains d'épices, de poids et de formes variés (cœur ou carré), aromatisés à la cannelle, à la muscade et au clou de girofle.

Le cas des pâtissiers est un bon exemple de métiers de bouche qui ont réussi à s'imposer dans le paysage urbain en cherchant à promouvoir une forme d'honorabilité nécessaire à l'exercice de leur profession. Ils répondaient avant tout à la demande des citadins dont un grand nombre se nourrissaient auprès des spécialistes du prêt à manger ${ }^{71}$. Ces spécialistes s'affirmèrent particulièrement dans la capitale du royaume, où les habitants faisaient appel à leurs services ${ }^{72}$. Une habitude que l'on rencontre déjà au $\mathrm{XIII}^{\mathrm{e}}$ siècle, comme en témoignent le Dictionarius de Jean de Garlande et

65. Sorte de beignet au fromage. «Talemose faicte de fin fromage par morceaulx carrés menu comme fèves, et parmy le fromage, soit destrampé œufz largement, et meslé tout ensemble, et la crouste destrampée d'œufz et de beurre » (Le Viandier, d'après l'édition de 1486, Pau, 2001, p. 40).

66. Les échaudés sont déjà cités dans les statuts des talemeliers du XIII ${ }^{\mathrm{e}}$ siècle comme étant confectionnés les jours de fêtes religieuses. Art. XXVIII : « Nus Talemeliers ne puet cuire au jour de la S. Jaque et S. Phelippe, ne au jour de la S. Denise, ne au jour de la Touz Sainz, ne au jour de la feste au[s] Mors, se ce ne sont eschaudés a doner por Dieu, ne au jour de la feste S. Genevieve après Noel » (Le Livre des métiers..., p. 9). L'échaudé est une pâte que l'on ébouillante avant de la passer au four.

67. «Lettres patentes de Charles IX confirmant les statuts communs aux deux métiers de pâtissiers et oubloyers, en trente-quatre articles » (juillet 1566) (Les Métiers et corporations..., p. 386-392).

68. «Premiers statuts des fabricants de pain d'épices, en vingt-sept articles, et lettres confirmatives de Henri IV (février 1596) (Les Métiers et corporations..., p. 400-404).

69. «Arrêt du Parlement rendu entre les pâtissiers simples ou de pain d'épices et les pâtissiers oubloyers au sujet de la visite des jurés » (7 septembre 1508) (Les Métiers et corporations..., p. 399).

70. P. Rambourg, « Sucre et mets sucrés dans les traités culinaires de la fin du Moyen Âge au début du XX' siècle », dans Du sucre, Biarritz, 2007, p. 173-188.

71. P. RAMBOURG, «Cuisine publique, cuisine de rue : espaces et modes de restauration » (chapitre IV), dans Histoire de la cuisine et de la gastronomie françaises, Paris, 2010, p. 6987.

72. Voir n. 10. 
Le Livre des métiers d'Étienne Boileau (avec les « cuisinier-oyers »), mais également les chroniques, les dits et les fabliaux ${ }^{73}$, et qui ne cessera de se conforter au fil du temps à travers la spécialisation des métiers de bouche.

Les charcutiers obtiennent leurs premiers statuts en 1476 pour la préparation des viandes cuites (principalement de porc) et des saucisses ${ }^{74}$. Les rôtisseurs, spécialisés dans l'apprêt des viandes rôties ou prêtes à rôtir, sont reconnus par lettres patentes en $1509^{75}$. Les sauciers, qu'une ordonnance de la fin du XIV ${ }^{\mathrm{e}}$ siècle associe aux vinaigriers et aux moutardiers, sont mentionnés dans les statuts des épiciers de 1450 où un article concerne les « espiciers et aultres personnes qui s'entremettront de faire et vendre saulces, a Paris, comme cameline, saulce vert, saulce rappée, saulce chaude, saulces a composte, saulce moustarde et aultre saulces ${ }^{76} »$, à savoir les sauces les plus populaires de l'époque ${ }^{77}$.

\section{La connaissance du métier}

Cette plus grande spécialisation des métiers de bouche, qui tend vers plus de qualité et vers une professionnalisation des pratiques culinaires et alimentaires, répond ainsi à une clientèle de plus en plus exigeante ${ }^{78}$. Mais cette évolution des métiers contribua dans le même temps à une fermeture progressive des corps professionnels, particulièrement au niveau de la maîtrise. L'accès à la maîtrise se faisait après un examen portant sur l'ensemble du métier, sur la connaissance théorique du candidat et sur l'élaboration d'un chef-d'œuvre montrant son habileté et son savoirfaire. La plupart des métiers adoptèrent cette pratique au cours des XIV et

73. À l'exemple du dit des «Trois dames de Paris » qui raconte le périple de trois Parisiennes qui décidèrent d'aller boire et manger à la taverne : Dits de Watriquet de Couvin, éd. A. SCHEler, Bruxelles, 1868, p. 381-390. Voir aussi D. AleXANDRE-Bidon et M.T. LorcIn, Le Quotidien au temps des fabliaux, Paris, 2003, p. 237.

74. «Lettres du prévôt de Paris qui homologuent les premiers statuts des charcutiers en dix-sept articles » (17 janvier 1476) (Les Métiers et corporations..., p. 319-323).

75. "Lettres patentes de Louis XII, homologuant les statuts des oyers rôtisseurs en 15 articles » (mars 1509) (Les Métiers et corporations..., p. 354-355).

76. «Lettres du prévôt de Paris présentant un nouveau texte de statuts, pour les épiciers, en 8 articles » (31 mars 1450) (Les Métiers et corporations..., p. 506-508).

77. Rappelons que la sauce médiévale a pour base un mélange d'épices délayées dans du vinaigre, du verjus ou du vin blanc.

78. Ainsi, si les « cuisiniers-oyers » des années 1260 disent apprêter des «viandes communes » pour le «peuple », les « queux-cuisiniers » du XVI ${ }^{\mathrm{e}}$ siècle s'adressent, quant à eux, à une clientèle plus élitiste comme le suggèrent leurs statuts : "Lettres patentes de Henri IV, confirmatives des premiers statuts des queux-cuisiniers porte-chappes, en douze articles » (mars 1599) (Les Métiers et corporations..., p. 303). 
$\mathrm{XV}^{\mathrm{e}}$ siècles ${ }^{79}$. S'ajoutait à cela la vénalité de la maîtrise, à l'exception de la gratuité consentie aux fils de maîtres.

Le futur maître devait aussi s'acquitter, «outre le prix d'achat du métier», d'une sorte de gratification qui pouvait porter le nom de past, d'abuvrement ou d'aboivrement. Le past était un repas que le nouveau membre offrait à ses confrères, l'abuvrement une collation. Cela se déroulait l'année de réception du nouveau membre ${ }^{80}$. Certains corps professionnels étaient très vigilants dans la transmission des savoirs et dans l'accessibilité à la maîtrise, à l'exemple des bouchers de la grande boucherie de Paris qui « n'admettaient dans leur sein que ceux qui y avaient droit par leur naissance $^{81} »$, les étals se transmettant de père en fils.

Chez les boulangers, l'aspirant à la maîtrise devait faire un « stage » de quatre ans après avoir acquis le métier. Durant cette période, le candidat donnait au roi une certaine somme d'argent à différents moments de l'année (Épiphanie, Pâques, Saint-Jean-Baptiste, Saint-Martin d'hiver) et « chaque semaine un tonlieu d'un denier et une obole de pain ${ }^{82} »$. Au terme de sa période probatoire, le candidat présentait au maître des boulangers un pot de terre neuf garni de noix et de «nieules », et lui déclarait qu'il avait fait ses quatre ans. Le maître s'assurait de l'exactitude de la déclaration auprès du percepteur, rendait le pot au candidat qui « le jetait contre le mur extérieur de la maison ». Tous célébraient ensuite le nouveau confrère autour d'une table.

L'oubloyer devait, en 1270, être capable de faire un millier de « nieles » par jour ${ }^{83}$; et en 1397 , cinq cents grandes oublies ${ }^{84}$, trois cents « supplications » (des sortes de gaufres) et deux cents « esterels » (qui

79. G. FagnieZ, Études sur l'industrie..., p. 93-94 ; B. GEREMEK, Le Salariat dans l'artisanat parisien aux XIII'-XVe siècles, Paris, 1992, p. 47.

80. G. FagniEZ, Études sur l'industrie..., p. 101.

81. Ibid., p. 103. «Lettres patentes de Charles VI portant confirmation des privilèges des bouchers de la grande boucherie et homologation d'un nouveau texte de statuts en quarantedeux articles» (juin 1381), art. 23 : «Item, nul ne peut estre bouchier de la grant boucherie de Paris, ne faire fait de bouchier ne de boucherie, se il n'est filz de bouchier de ycelle boucherie » (Les Métiers et corporations..., p. 266-272). Voir aussi J. BOUSSARD, De la fin du siège de 885-886 à la mort de Philippe Auguste, Paris, 1996, p. 299-301 ; et B. DESCAMPS, «Tuer, tailler et vendre char »: les bouchers parisiens à la fin du Moyen Âge, v. 1350-v. 1500, thèse, Université Paris 1-Panthéon-Sorbonne, 2009.

82. G. FaGnIEZ, Études sur l'industrie..., p. 102-103. Au commencement de chaque année, une coche était faite sur la taille du percepteur qui libérait l'aspirant des redevances de l'année précédente. «Cis titres parole des Talemeliers qui sunt dedens la banliue de Paris » (Le Livre des métiers..., p. 3-15).

83. " Lettres de Regnaut Barbou, prévôt de Paris, contenant les statuts des oubloyers ou fabricants d'oublies dépendant de la communauté des pâtissiers, en onze articles » (mai 1270) (Les Métiers et corporations..., p. 369-371). Les « nieles » ou «nieules » étaient des sortes de pâtisseries faites d'une pâte au levain ébouillantée et séchée au four.

84. Des sortes de gaufrettes ornées de symboles religieux. 
sont aussi des espèces d'oublies), et savoir confectionner sa propre pâte ${ }^{85}$. Neuf ans plus tard un nouveau règlement oblige les maîtres à ne prendre qu'un seul apprenti pour une durée d'au moins cinq ans, sous prétexte que le métier d'oubloyer « est bien dangereux et subtil à apprendre ». Le prétendant à la maîtrise doit être fils de maître et faire un chef-d'œuvre pour pouvoir tenir un ouvroir ${ }^{86}$.

Pour les charcutiers (statuts de 1476), « nul homme ne pourra estre maistre saulsissier et charcuitier, cuire char, faire saulcisses, ne tenir ouvrouer, ne fenestre ouverte a Paris, s'il n'a esté quatre ans apprentiz a maistre dudit mestier, a Paris, et fait chef d'euvre, ou s'il n'est expert oudit mestier et tel rapporté par les jurez et fait chef d'euvre ${ }^{87} »$. Et les maîtres queux, cuisiniers et porte-chapes rappelaient aux candidats à la maîtrise que le chef-d'œuvre devait être fait « de chair et de poisson, le tout diversement et a ses depens, selon les saisons de l'année et sera fait en la maison de l'un desdits jurez, auxquels pourront assister douze maistres dudit mestier ${ }^{88}$ » (mars 1599). Cette évolution de la réglementation des corps professionnels qui développe les «privilèges corporatifs ${ }^{89}$ » et la limitation d'accès aux métiers, renforce, d'une certaine manière, les savoir-faire et les domaines de compétences.

Car présenter un chef-d'œuvre suppose la connaissance du métier. Celle-ci se définit d'abord par une période de formation et d'apprentissage. « L'apprentissage est de règle dans les métiers parisiens ${ }^{90}$ », et d'une façon générale les statuts renseignent sur les conditions de l'apprentissage, sur sa durée, qui est variable selon les métiers, avec toutefois une tendance à l'allongement du temps de formation. Ces textes donnent aussi des informations sur le nombre d'apprentis « étrangers ${ }^{91}$ » par atelier,

85. « Lettres du prévôt de Paris contenant les lettres patentes de Charles VI du 9 septembre 1397, la requête des oubloyers avec sept articles d'anciens statuts et une nouvelle rédaction de statuts en treize articles » (18 octobre 1397), art. 1 : «Premièrement, que nul ne puisse tenir ouvrouer ne estre ovrier en la ville de Paris, ne ès forbours, s'il ne scet faire en un jour, au moins cinq cens de grant oublées, trois cent de supplicacions et deux cent d'esterels du mestier, bons et souffisans, et faire sa paste pour ledit ouvrage » (Les Métiers et corporations..., p. 371-374).

86. «Lettres patentes de Charles VI, portant confirmation des règlements précédents des pâtissiers-oubloyers et addition de six nouveaux articles » (août 1406), art. 12 : «Item, aucun dudit mestier ne pourra tenir ouvrouer d'icellui, s'il n'est filz de maistre, jusques ad ce qu'il ait fait son chief d'euvre et qu'il soit approuvé souffisant par les maistres dudit mestier » (ibid., p. 374-376).

87. «Lettres du prévôt de Paris qui homologuent les premiers statuts des charcutiers en dix-sept articles » (17 janvier 1476) (ibid., p. 319-323).

88. «Lettres patentes de Henri IV, confirmatives des premiers statuts des queux-cuisiniers porte-chappes, en douze articles » (mars 1599) (ibid., p. 303-305).

89. B. GEREMEK, Le Salariat..., p. 45.

90. Ibid., p. 30.

91. Qui n'appartient pas à la famille de l'artisan. 
généralement un à deux, pour garantir, entre autres, la « qualité technique de l'enseignement », mais pas seulement ${ }^{92}$.

Après l'acquisition des connaissances de base, l'apprenti qui a fini son temps d'apprentissage parfait son savoir-faire et renforce son expérience professionnelle en travaillant dans des ateliers. Il devient un ouvrier qualifié que les textes nomment généralement «valet », suggérant ainsi son statut de subordonné. Nous entrons là dans la hiérarchie professionnelle des métiers qui distingue trois principaux niveaux de compétence : l'apprenti, le valet (que l'on appellera progressivement compagnon) et le maître. Le valet passe alors un contrat de louage avec un maître qui lui donne un salaire en échange de son travail ${ }^{93}$. Parfois les règlements soulignent la méconnaissance professionnelle que pourraient avoir des fils de maître. Ils doivent alors recevoir une formation, comme le proposent les statuts des « cuisiniers-oyers » des années $1260^{94}$ :

Item, que nulz ne puisse prendre varlet ou dit mestier d'ores en avant, se il n'a esté aprentiz oudit mestier deux ans ; ou se il n'est filz de mestre et aucune chose sache oudit mestier. Et se le filz du mestre ne sait riens du mestier par quoi il puisse la marchandise exercer, que il tiengne a ses despens un des ouvriers dudit mestier qui en soict expers jusques a tant que ycelui fils de maistre le sache convenable exercer aus diz des maistres dudit mestier.

Si les textes réglementaires s'attachent à définir l'organisation professionnelle du métier concerné, à combattre les mauvaises pratiques, ils peuvent aussi livrer des informations sur la connaissance technique à acquérir et sur des manières de faire. Certes, les sources normatives ne constituent pas des recueils de recettes, mais la tendance qui veut que l'on nomme précisément les mets confectionnés, comme nous avons pu le constater chez les pâtissiers des $\mathrm{XV}^{\mathrm{e}}$ et $\mathrm{XVI}^{\mathrm{e}}$ siècles, suggère des pratiques culinaires et des recettes que l'on retrouve dans les traités de cuisine ${ }^{95}$, à l'exemple des sauces citées dans les statuts des épiciers de 1450. Ces règlements distinguent les savoir-faire normés de ceux qui ne le sont pas, et affirment les domaines de compétence

92. Pour Bronislaw Geremek, le contrôle du nombre d'apprentis sert également à limiter l'afflux de nouveaux artisans et à empêcher une trop grande concurrence.

93. En théorie, tous les valets pouvaient accéder à la maîtrise ; en pratique la situation était beaucoup plus compliquée.

94. «Des cuiseniers », art. II, Le Livre des métiers..., p. 145-147.

95. Sur les traités de cuisine français, voir B. LAURIOUX, Le Règne de Taillevent. Livres et pratiques culinaires à la fin du Moyen Âge, Paris, 1997. 
du professionnel, bannissant « les autres de mestier honteux et deshonneste ou repugnant a icelluy mestier, ou autres hazardeurs ${ }^{96} »$.

Codifier des pratiques alimentaires, c'est en même temps construire l'identité d'un métier, en le positionnant par rapport aux autres. Ce n'est ainsi pas un hasard si les charcutiers donnent la recette de la saucisse dans leurs premiers statuts de $1476^{97}$. La confection des saucisses les démarque des cuisiniers et des rôtisseurs, mais aussi des regrattiers, car les charcutiers ne devaient «vendre aucuns fruiz, choux, porrées, verdures, navetz, beurres, fromages et autre choses, excepté saulsisses, chars cuites, saindoulx et autres chars et denrrée de boucherie qu'ilz ont accoustumé vendre », précise l'article 5 de leurs statuts. Les charcutiers affirmaient ainsi leurs prérogatives, officialisaient leur savoir-faire, et confortaient du même coup une conscience professionnelle, avec notamment la reconnaissance de leur règlement de métier. On retrouve la même volonté identitaire dans les premiers statuts des fabricants de pain d'épices ${ }^{98}$, à l'extrême fin du $\mathrm{XVI}^{\mathrm{e}}$ siècle, mais aussi dans ceux des maîtres-queux et des cuisiniers qui rappellent à leurs collègues pâtissiers, rôtisseurs et charcutiers, qu'ils sont les seuls habilités à œuvrer lors des noces, festins et banquets ${ }^{99}$. Tout cela suggère des conflits récurrents entre les corps de métier qui cherchent à délimiter leurs professions pour mieux se distinguer les uns des autres et affirmer les savoir-faire qui garantissent leur existence respective.

Ainsi, partis de la nécessité de nourrir les Parisiens, objectif premier des métiers de l'alimentation, et arrivant peu à peu à une plus grande spécialisation de leurs pratiques culinaires et alimentaires, les artisans de bouche affirmèrent sur la longue durée le caractère professionnel de leur métier. La clientèle qui faisait appel à leur service ne le faisait pas seulement pour s'alimenter, mais aussi parce que ces professionnels étaient

96. «Lettres de Jacques d'Estouteville, prévôt de Paris, contenant une requête des pâtissiers, avec sept articles de statuts » (6 juin 1497) (Les Métiers et corporations..., p. 383385).

97. «Lettres du prévôt de Paris qui homologuent les premiers statuts des charcutiers en dix-sept articles » (17 janvier 1476), art. 10 : « Item que nul ne face saulcisses, sinon de char de porc fraiz, haiché bien menu, a ce que la char preigne mieulx le sel, que ladicte char soit bien salée de menu sel, et que en icelles ne soit mis avecques ladite char et sel, sinon du fanoul qui soit bon, nect et bien esleu, et qu'il ne sente le viel, le moisy, ne autre goust, et que lesdites saulcisses ne soient couvertes, sinon de menuz boyaulx de porc, bons et dignes de user a corps humain, sans y applicquer autres boyaulx [...]» (ibid., p. 319-323).

98. «Premiers statuts des fabricants de pain d'épices, en vingt-sept articles, et lettres confirmatives de Henri IV » (février 1596) (ibid., p. 400-404).

99. «Lettres patentes de Henri IV, confirmatives des premiers statuts des queux-cuisiniers porte-chappes, en douze articles » (mars 1599), art. 1 : « Premierement, que les paticiers, rotisseurs, chaircuitiers et autres personnes, de quelque mestier qu'ils soient, ne pourront entreprendre dudit mestier pour faire nopces, festins ou banquets, tant en leurs maisons qu'en autres lieux, si ce n'est chacun de leur mestier » (ibid., p. 303-305). 
des spécialistes du «prêt-à-manger »: ils fournissaient une nourriture cuisinée et certains d'entre eux avaient acquis une belle renommée ${ }^{100}$. Ces spécialistes surent en effet conforter et préciser leur savoir-faire et leur technique, pour déterminer une bonne pratique et définir les spécificités de leurs métiers. Cette évolution s'inscrit dans une tendance générale qui touche « tous les grands secteurs industriels ${ }^{101}$ ». Cela ne se fit pas sans friction entre les différentes communautés. Mais l'affirmation et la spécialisation des professions de bouche attestent de leur réussite dans la ville et d'un art du prêt à manger déjà bien développé ${ }^{102}$.

\section{Patrick Rambourg - Université Paris Diderot-Paris 7 - ICT}

\section{Pratiques alimentaires, savoir-faire et professionnalisme dans les métiers de bouche parisiens (fin du Moyen Âge et Renaissance)}

À partir de textes réglementaires, cet article montre comment les métiers de bouche se sont affirmés dans le Paris de la fin du Moyen Âge et de la Renaissance. II insiste plus précisément sur les spécialistes du " prêtà-manger " qui, au fil du temps, ont conforté leurs savoir-faire, leurs compétences techniques et les contours de leurs spécialisations, pour construire leurs identités professionnelles.

Alimentation - métier de bouche - Paris - prêt-à-manger - savoir-faire

\section{Food Practices, Know-How and Professionalism in the Parisian Food Trades (Late Middle Ages and Renaissance)}

Based on statutory texts, this article shows how food trades asserted themselves in the Paris of the late Middle Ages and the Renaissance. It focuses specifically on specialists of "ready-to-eat ", which, over time, have reinforced their know-how, their technical skills, and the contours of their specializations, to build their professional identities.

Food - food trade - know-how - Paris - ready-to-eat

100. Le Viandier de la bibliothèque Vaticane (1450-1460) souligne la réputation des « oyers » (ceux qui rôtissent des oies) du quartier Saint-Merri, du carrefour Saint-Severin et de la Porte Baudoyer (Le Viandier de Guillaume Tirel dit Taillevent, éd. J. PICHON et G. VICAIRE, Paris, 1892, p. 227).

101. «Travail du textile, des cuirs et peaux, des métaux ou du bois » écrit F. Desportes dans «Les métiers de l'alimentation » (dans J-L. Flandrin et M. MONTANARI, Histoire de l'alimentation, Paris, 1996, p. 439).

102. En témoigne l'ambassadeur Jérôme Lippomano dans les années 1570, lors de son séjour à Paris : « [...] les bouchers, les marchands de viande, les rôtisseurs, les revendeurs, les pâtissiers, les cabaretiers, les taverniers s'y trouvent en telle quantité que c'est une vraie confusion. [...] Voulez-vous votre provision toute prête, cuite ou crue ; les rôtisseurs et les pâtissiers en moins d'une heure vous arrangent un dîner, un souper pour dix, pour vingt, pour cent personnes : le rôtisseur vous donne la viande, le pâtissier les pâtés, les tourtes, les entrées, les desserts ; le cuisinier vous donne les gelées, les sauces, les ragoûts » (M. N. TOMMASEO, Relations des ambassadeurs vénitiens sur les affaires de France au XVI siècle, Paris, 1838, t. II, p. 601-603). 